\title{
Recurrent pneumomediastinum in a patient with rheumatoid arthritis
}

\author{
H. Bhardwaj', B. Bhardwaj', P.V. Carlile 1
}

\begin{abstract}
Recurrent pneumomediastinum in a patient with rheumatoid arthritis. H. Bhardwaj, B. Bhardwaj, P.V. Carlile.

Spontaneous pneumomediastinum (SPM); also known as mediastinal emphysema, is a rare and usually benign self-resolving appearance of extraluminal air in the mediastinum without any underlying trigger. This is an uncommon disorder mostly seen in the young males and classic clinical presentation is with chest pain, dyspnea, cough and appearance of subcutaneous emphysema. Al-
\end{abstract}

\begin{abstract}
though several connective tissue disorders have been reported in association with SPM, it is a rare occurrence in rheumatoid arthritis (RA) with only small number of cases reported in literature. We report a 69 years old male with RA who developed recurrent asymptomatic episodes of SPM detected over a period of one year. The recurrent but benign episodes of SPM in this patient reestablish the usual uncomplicated course of this unusual clinical entity even in the rare recurrent cases.

Monaldi Arch Chest Dis 2013; 79: 3-4, 136-139.
\end{abstract}

Keywords: Spontaneous pneumomediastinum, Rheumatoid Arthritis, Interstitial Lung Diseases.

1 Pulmonary Medicine \& Critical Care, Internal Medicine, University of Oklahoma Health Sciences Center, Oklahoma City, USA.

2 Pulmonary Medicine and Tuberculosis, Internal Medicine, Indira Gandhi Medical College Shimla, India.

Correspondence: Himanshu Bhardwaj MD, Pulmonary and Critical Care Medicine, Department of Medicine/OUHSC, PO Box 26901,WP1310,Oklahoma City,OK 73190,U.S.A.; e-mail: himanshu-bhardwaj@ouhsc.edu

Spontaneous pneumomediastinum (SPM) is defined as the appearance of extraluminal air within the mediastinum without any underlying trigger or cause. It is rare disorder, mostly seen in young males and it usually has a benign self-limiting clinical course. SPM has been described in association with different connective tissue diseases with most of the cases reported in dermatomyositis patients. SPM secondary to rheumatoid arthritis (RA) related interstitial lung disease (ILD) is very rare and only handful of cases have been reported in the literature. We report a patient of RA related ILD who presented with recurrent episodes of SPM; each of these episodes was managed conservatively without any complication. Besides the rarity of SPM reported in RA, another unusual aspect in our presented case here is the recurrent nature of SPM. An overview of SPM with different proposed pathophysiological mechanisms and management considerations are discussed.

\section{Case Report}

A 69 years old Native American male with known history of rheumatoid arthritis (rheumatoid factor positive and anti-CCP antibody positive) and interstitial lung disease (ILD) related to rheumatoid arthritis was evaluated in our chest medicine clinic with 2 weeks history of worsening dyspnea and worsening cough of the same duration. He denied any fever, chills or night sweats. At baseline patient's pulmonary function tests were consistent with moderate restriction with $\mathrm{FVC}$ measured at $57 \%, \mathrm{FEV}_{1}$ of $66 \%, \mathrm{FEV}_{1} / \mathrm{FVC}$ ratio of $89 \%$ and DLCO of $29 \%$. His medications for rheumatoid arthritis included prednisone 5 $\mathrm{mg} /$ day, sulphasalazine and infliximab. In the clinic, patient was in mild respiratory distress due to dyspnea; his vital signs included blood pressure $110 / 70$, respiratory rate 20 , pulse 92 and temperature 99 degree Fahrenheit. He was saturating 93\% on his home oxygen through nasal cannula at 3 liters/minute. Significant physical examination findings were consistent with bibasal velcro-type crackles on lung auscultation. Hamman's crunch was not heard on cardiac auscultation. There was no neck vein distention or subcutaneous emphysema. A plain chest radiograph was done which showed bilateral extensive generalized fibrosis, most pronounced in the lung bases consistent with his established diagnosis of RA related ILD (figure 1). No mediastinal extraluminal air was seen on the plain chest radiograph. Due to worsening dyspnea and cough in past 2 weeks and history of significant ILD, we obtained a computed tomographic scan of the chest which showed large amount of pneumomediastinum which extended from the level of the aortic arch inferiorly, to below the level of the carina (figure 2 Panel A). Patient was admitted to the hospital for observation and was started on cough suppressant medications. Basic laboratory tests including complete blood count and chemistry panel did not show any abnormality. A barium swallow was done to rule out esophageal per- 


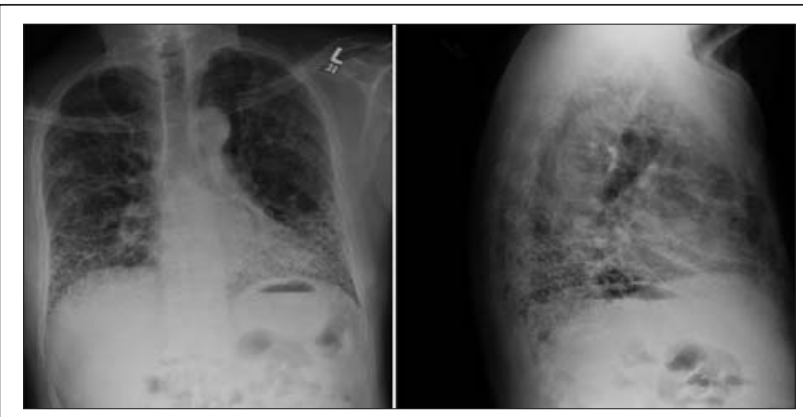

Fig. 1. - Chest Radiograph PA and Lateral view, showing extensive bibasilar fibrosis with relative sparing of the apices consistent with RA associated ILD.

foration which did not show any leak. Over next three days in the hospital, patient's cough as well as dyspnea improved significantly and he was discharged home in stable condition. A follow up CTScan of the chest 3 months later showed resolution of SPM (figure 2 Panel B).

Patient was followed in our pulmonary medicine clinic and over next one year he got one more CT-Scan of the chest for unrelated reason (for follow up of the lung nodules) which surprisingly, again showed new onset SPM, although in lesser amount compared to previous one (figure 2 Panel C). Since patient was completely asymptomatic, we decided not to pursue any intervention and only follow patient in the clinic with close observation. Patient has remained asymptomatic till date.

\section{Discussion}

Pneumomediastinum, also known as mediastinal emphysema is defined as the presence of extraluminal air within the mediastinum. First ever description of this clinical entity in the medical literature was by Laennec [1] in 1827. Pneumomediastinum can be classified into two major forms based on etiology: traumatic and spontaneous. Traumatic forms of pneumomediastinum are much more encountered and usually result from thoracic trauma and iatrogenic injuries to respiratory \& digestive tracts. Spontaneous pneumomediastinum (SPM) is defined as that occurring without any apparent triggering event. SPM was first described by Louis Hamman [2] in 1939 with the description of classic physical examination sign named after his name 'Hamman's crunch' described as 'crunching rasping sound which can be heard in synchrony with heart beats' during auscultation in some pneumomediastinum patients. SPM is an uncommon disorder with overall incidence ranging from $0.003 \%$ to $0.0095 \%$ in all hospitalized patients based on 3 major case series reported so far [3-5]. Most commonly it is seen in young male adults with age distribution between 18 years to 25 years. In the largest review published so far, Takada et al [6] described total 414 patients of SPM described in various major case series, $303(73.1 \%)$ patients in this review were males.
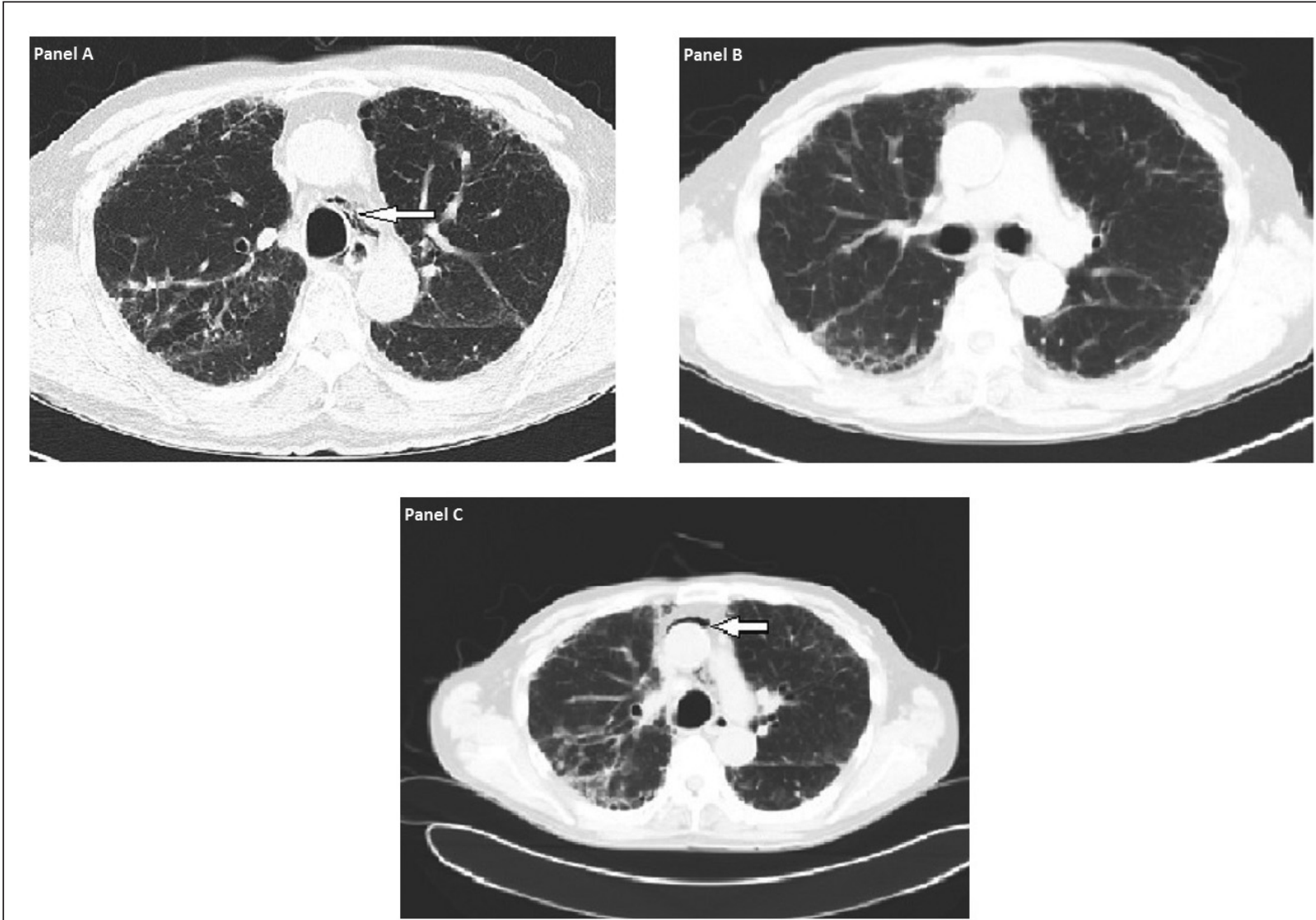

Fig. 2. - Panel A: CT-Scan chest at the time of diagnosis, showing significant pneumomediastinum with air tracking along the bronchovascular bundles (Arrow). Panel B: CT-Scan chest 3 months after the diagnosis, showing complete resolution of the pneumomediastinum. Panel C: CT-Scan chest at 1 year after the diagnosis, showing recurrent pneumomediastinum with air around the aorta (Arrow). 
The pathophysiological mechanisms underlying the development of SPM are based on "pressure gradient' theory described by Macklin \& Macklin in 1939 with animal model experiments, this theory is also known as 'Macklin Effect' [7]. The theory explains the development of SPM based on the index event of an acutely raised intraalveolar pressure causing increase in the pressure gradient between the air-filled alveoli and their surrounding interstitial space and eventually resulting in alveolar rupture. Some of the precipitating events known to increase the intraalveolar pressure and possibly predispose to the development of SPM include the factors that provoke Valsalva maneuver: coughing, sneezing, vomiting, child birth and inhalation of drugs (speed, cocaine, amphetamine-derived drugs). After the alveolar rupture, the free gas dissect along the peribroncho - vascular fascial sheaths into the hilum and then into the mediastinum resulting in SPM. Off note, once in the mediastinal space, the air can extend further through the fascial planes connecting the cervical soft tissues with the mediastinal, retroperitoneal spaces and may even extend upward to the prevertebral space.

Various connective tissue diseases related interstitial lung diseases (ILD) have been reported to be associated with SPM. Most commonly it is seen in polymyositis/dermatomyositis patients with overall prevalence of around $8.3 \%$ in this patient population [8]. Others have reported SPM in lupus [9-11], systemic sclerosis [12-14], and also rheumatoid arthritis [15-17]. In one recent large review, Goff et al [18] reported total 62 patients of connective tissue diseases associated SPM described in literature so far and $49(79 \%)$ of these patients had dermatomyositis. There were three patients each of polymyositis, lupus, systemic sclerosis and rheumatoid arthritis (4.8\% each). For rheumatoid arthritis related SPM, besides our presented case, one other case reported had persistent SPM like our patient [15].

Clinical symptoms in SPM can be diverse and vary depending on the amount of trapped air and the inflammation in adjacent mediastinal tissues. Significant numbers of patients remain asymptomatic but chest pain, cough and dyspnea are the most commonly seen symptoms. Iyer et al [19] reported a $63 \%$ prevalence of chest pain, $45 \%$ of dyspnea and $44 \%$ of cough in a retrospective review of total 62 patients with SPM. The typical chest pain in SPM patients is known to radiate towards back \& neck and it gets exacerbated with swallowing, inspiration or leaning forward. Some other reported symptoms include neck pain, dysphagia, dysphonia and dizziness. Like clinical symptoms, physical examination findings in SPM patients are diverse too. The most remarkable physical examination finding is subcutaneous emphysema which was reported in $62 \%$ cases of the total 414 SPM patients reviewed by Takada et al [6]. Most popular auscultative finding in SPM remains 'Hamman's crunch' described as loud bubbling crunchy sound heard with each heart beat. Although popular, prevalence of Hamman's crunch in SPM patients is only around 30\% [6]. Other less commonly reported signs include the presence of pulsus paradoxus, cyanosis, and muffled heart sounds [3].

The diagnosis of SPM is made based on the different radiographic findings. Chest radiographs are the initial diagnostic tests of choice and for definitive diagnosis computed (CT) scan of the chest is the preferred test. On chest radiographs, some of the common suggestive findings include the presence of lucent streaks of gas outlining mediastinal structures, demonstration of subcutaneous emphysema and prominence of the cardiac silhouette. Some of the other infrequently seen chest radiographic signs are the double-bronchial-wall sign, continuous diaphragm sign, Naclerio's V sign and the-ring-around-the-artery sign. Although, chest radiographs remain useful diagnostic tests in the diagnosis of SPM, some of the cases may be missed with plain chest radiographs. Hence, chest CT scan is thought to be the most reliable diagnostic modality in diagnosing SPM. Furthermore, chest CT may reveal undiagnosed pre-existing pulmonary disease. Some of the additional diagnostic tests performed in SPM patients to investigate the cause of air leakage after the initial diagnosis is made include esophagoscopy, esophagram or flexible bronchoscopy.

Most of the uncomplicated cases of SPM are self-limiting and resolve on its own; only watchful observation with adjunctive analgesic treatments is needed. Multiple reports in the literature recommend hospital admission for observation. Other treatment approach reported in several other reports involves the breathing of $100 \%$ of oxygen which is thought to promote rapid reabsorption of the mediastinal air by so called 'nitrogen washout' method [15]. Although a proven treatment in the management of pneumothorax, the efficacy of this therapeutic intervention in cases of SPM is not conclusive and routine use is not recommended [4].

Overall clinical course in SPM patients is usually benign with very low rate of complications. Most of the symptomatic patients show improvement in the symptoms within 2 days and resolution of the radiographic findings within one week [6]. The recurrence of SPM after initial resolution is also rare; in the review by Takada [6], only five $(1.2 \%)$ out of total 414 SPM patients were found to have recurrence. Natale et al [21] also described 12 cases of recurrent pneumothoraces described in various case series but they were able to demonstrate a precipitating cause for recurrent SPM in most of the patients. Persistent and prolonged SPM of weeks to month's duration like in our presented case is also very rare and it is usually seen in patients with pre-existing underlying lung diseases. One such case of RA associated SPM of 2 months duration was reported by Patel et al [15]. Normally the management of the recurrent episode is similar to that of the first episode, i.e. rest, cough suppressants and analgesia.

We conclude that SPM is a rare and unusual complication of connective tissue related lung dis- 
eases. Most of the cases have been reported in association with dermatomyositis, although scattered reports have shown development of SPM in some other connective tissue diseases too. To our knowledge, our presented case represents the fourth such case of SPM seen in association with RA. Recurrent course seen in our patient makes it more unusual and probably represents persistent air leak in terminal alveoli. Regardless of the underlying cause, the benign course and self-resolution of the SPM with only watchful observation in our patient reestablishes the usual uncomplicated nature of this clinical entity even in the rare recurrent cases.

\section{References}

1. Laennec RT. A treatise on the diseases of the chest and on mediate auscultation. 3rd ed. London: Thomas \& George Underwood; 1829. p. 152-78.

2. Hamman L. Spontaneous mediastinal emphysema. Bull Johns Hopkins Hosp 1939; 64: 1-21.

3. Abolnik I, Lossos IS, Breuer R. Spontaneous pneumomediastinum: a report of 25 cases. Chest 1991; 100: 93-5.

4. Takada K, Matsumoto S, Hiramatsu T, et al. Management of spontaneous pneumomediastinum based on clinical experience of 25 cases. Respir Med 2008; 102: 1329-34.

5. Kim DH, Park JH, Chei CS, et al. Spontaneous pneumomediastinum: clinical investigation. Korean J Thorac Cardiovasc Surg 2006; 39: 220-5.

6. Takada K, Matsumoto S, Hiramatsu T, et al. Spontaneous pneumomediastinum: an algorithm for diagnosis and management. Ther Adv Respir Dis 2009; 3: 301-7.

7. Macklin MT, Macklin CC. Malignant interstitial emphysema of the lungs and mediastinum as an important occult complication in many respiratory diseases and other conditions: an interpretation of the clinical literature in the light of laboratory experiment. Medicine 1944; 23: 281-358.

8. Kono H, Inokuma S, Nakayama H, Suzuki M. Pneumomediastinum dermatomyositis: association with cutaneous vasculopathy. Ann Rheum Dis 2000; 59: 372-6.
9. Richards AJ, Talbot IC, Swinson DR, Hamilton EB. Diffuse pulmonary fibrosis and bilateral pneumothoraces in systemic lupus erythematosus. Postgrad Med J 1975; 51: 851-5.

10. Masuda A, Tsushima T, Shizume K, et al. Recurrent pneumothoraces and mediastinal emphysema in systemic lupus erythematosus. J Rheumatol 1990; 17: 544-8.

11. Paira SO, Roverano S. Bilateral pneumothorax and mediastinal emphysema in systemic lupus erythematosus: Clin Rheumatol 1992; 11: 571-3.

12. Mohammad A, Boon Low T, O'Dwyer D, et al. Spontaneous pneumo-mediastinum in systemic sclerosis: a case report. Rheumatology (Oxford) 2007; 46: 1376-7.

13. Teixeira Moreira Almeida Mdo S, Dias LT, Fernandes SJ, et al. Spontaneous pneumomediastinum and subcutaneousemphysema in systemic sclerosis. Rheumatol Int 2007; 27: 675-7.

14. Jun JB, Song SY. The development of pneumomediastinum after pulmonary function testing in a patient with systemic sclerosis [letter]. Rheumatol Int 2007; 27: 1097-8.

15. Patel A, Kesler B, Wise RA. Persistent pneumomediastinum in interstitial fibrosis associated with rheumatoid arthritis: treatment with high-concentration oxygen. Chest 2000; 117: 1809-13.

16. Dikensoy O, Bayram N, Bingol, et al. Bronchiolitis obliterans in a case of juvenile rheumatoid arthritis presented with pneumomediastinum. Respiration 2002; 69: 100-2.

17. Kobayashi T, Satoh K, Ohkawa M, et al. Multiple rheumatoid nodules with rapid thin-walled cavity formation producingpneumothorax. J Thorac Imaging 2005; 20: 47-9.

18. Le Goff B, Chérin P, Cantagrel A, et al. Pneumomediastinum in interstitial lung disease associated with dermatomyositis and polymyositis. Arthritis Rheum 2009 15; 61: 108-18.

19. Iyer VN, Joshi AY, Ryu JH. Spontaneous pneumomediastinum: analysis of 62 consecutive adult patients. Mayo Clin Proc 2009; 84: 417-21.

20. Bejvan SM, Godwin JD. Pneumomediastinum: old signs and new signs. AJR Am J Roentgenol 1996; 166: 1041-8.

21. Natale C, D'Journo XB, Duconseil P, et al. Recurrent spontaneous pneumomediastinum in an adult. Eur $J$ Cardiothorac Surg 2012; 41: 1199-201.

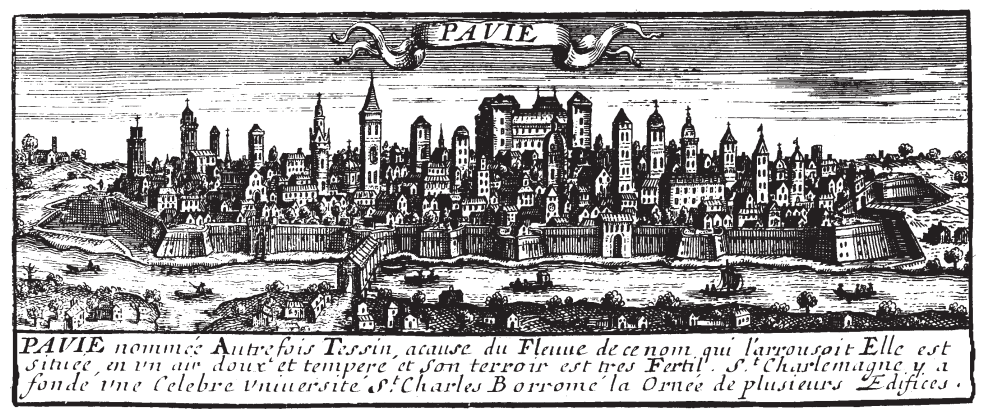

